Objectives: The aim of this study was to determine possible correlation of the SSc clinical parameters with oral health-related quality of life measured with the Oral Health Impact Profile 49 (OHIP 49).

Methods: Subjects were recruited from the Center of excellency for systemic sclerosis in Croatia cohort. Detailed dental by the same dentist and clinical examinations were performed according to standardized protocols. Associations between oral health-related quality of life and disease charachteristics were examined. We evaluated the disease severity using clinical and laboratory parameters according to the Medsger Severity Scale. The level of SSc activity was evaluated according to Valentini activity score. Oral quality of life was measured using the OHIP 49, which consists of 49 questions on the frequency of adverse oral conditions such as toothache, mouth pain, difficulty chewing or pronouncing words and discomfort related to appearance (higher scores indicating worse oral health-related quality of life). The study was approved by the University Hospital Split Ethics Committee.

Results: Thirty-one SSc patients (29 women and 2 men, mean age $56.45 \pm 13.60$ years, median disease duration 7 years with minimum-maximum range 1-28 years) were consecutively enrolled for this study between January 2014 and December 2015. All patients fulfilled the ACR criteria for the diagnosis of SSc. The distinction between limited cutaneous SSc (IcSSc) and diffuse cutaneous SSc (dcSSc) was made according to the Leroy et al. criteria (28 dcSSc, $3 \mathrm{lcSSc}$ ). OHIP 49 scores highly positively correlated with disease activity $(p=0.005, r=0.4872$, Spearman's rank coefficient) and severity $(p=0.016, r=0.4303$, Spearman's rank coefficient). Furthermore, oral health-related quality of life positively correlated with the skin involvement evaluated by modified Rodnan skin score $(p=0.003$, $r=0.5207$, Spearman's rank coefficient). Impaired quality of oral health positively correlated with the severity of general involvement, skin, gastrointestinal and joint/tendon involvement $(p=0.003, r=0.506$ for general involvement, $p=0.003$, $r=0.511 ; p<0.001, r=0.591$ and $p=0.02, r=0.391$ for skin, gastrointestinal and joint/tendon involvement, respectively, Spearman's rank coefficient). OHIP 49 score was highly variable between anti-topoisomerase I antibodies positive or negative SSc patients ( $p<0.001$, Fisher's exact test).

Conclusions: Contrary to previous studies in our study disease severity and activity were related to OHIP 49 scores. Our data suggest that OHIP scores correlate with severity of general involvement, skin, gastrointestinal, and joint/tendon involvement in SSc patients. Disease subset and autoantibodies profil could play a role in the oral manifestation of SSc. Better collaboration between rheumatologists and the dental team is required to improve access to dental care and oral health outcomes for SSc patients.

Disclosure of Interest: None declared

DOI: 10.1136/annrheumdis-2017-eular.6267

\section{AB0662 SYSTEMIC SCLEROSIS (SSC) COHORT IN ABU DHABI: FOCUS ON DIGITAL ULCERS (DU)}

M. Al-Maini ${ }^{1}$, H. Al-Mashari ${ }^{1}$, T. Khan $^{1}$, N. Abutaha ${ }^{1}$, R. Aneja ${ }^{1}$, A. Las Alas ${ }^{1}$, S. Gonuguntla ${ }^{1}$, M. Cerinic ${ }^{2}$, K. Khawaja ${ }^{1} .{ }^{1}$ Adult and Pediatric Rheumatology, Allergy and Immunology, Al-Mafraq Hospital, Abu Dhabi, United Arab Emirates; ${ }^{2}$ Rheumatology, University of Florence, Florence, Italy

Background: DU are a significant burden for SSc patients affecting approximately $40-50 \%$ of patients (1). No data are available on the frequency of DU on SSC patients living in the United Arab Emirates.

Objectives: To identify the frequency of DU in a cohort of SSC in Abu Dhabi which is the capital city of the United Arab Emirates (UAE).

Methods: SSC patients identified according to ACR EULAR criteria through the hospital electronic medical records system, which was implemented from January 2011 for medical documentation in all public hospitals in Abu- Dhabi and districts. Using the International statistical classification of diseases and related health problems, usually called by the short form name international classification of Diseases (ICD), version 9 (710.1) from 1st of January 2011 to 31st of December 2016. Retrospective review of electronic medical records and paper case notes was performed on patients who presented during this period. The frequency of DU was searched. DU were defined (1) and categorised (2) (no digital ulcers, episodic (rarely recurrent), recurrent (frequently recurrent) and chronic ( $\geq 1 \mathrm{DU}$ at every follow up). Results and the incidence of gangrene were compared to data from DUO registry (3).

Results: 47 SSc patients (Male to female ratio 12 (25.5\%) to 35 (74.5\%) (Duration of disease from 2 to 24 years with peak age for ulcers between $40-50$ years) were identified. No ulcers were detected in 34 patients $(72.3 \%)$ while DU were found in 13 patients (27.7\%): they were episodic in 9 patients (19\%) and chronic in 4 patients $(8.5 \%)$ of the total patients. No recurrent ulcers were found. Only one patient $(2 \%)$ evolved to gangrene (she was a smoker). The other 12 patients with DU were non-smokers and three of them were under 16 years of age.

Conclusions: In Abu Dhabi SSc population, the incidence of DU is $27.7 \%$. This differs from the incidence in the western literature where it peaks from 40 to $50 \%$ (3). Our patients had episodic (19\%) and chronic DU $(8.5 \%)$ of the total patients. The incidence of gangrene in our cohort was lower when compared to the data reported in the DUO registry (20\%) (2). Although patients live in a very warm climate, DU are still experienced in SSc in Abu Dhabi. Likely, the chronic exposure to air conditioning may explain this paradox.

References:

[1] Suliman $Y$ et al. Preliminary musculoskeletal ultrasound (MSUS) ulcer definition does not correlate with visual observation in systemic sclerosis (SSc) patients. J. scleroderma relat. disord. 2017 (in press).

[2] Matucci-Cerinic $\mathrm{M}$ et al. Elucidating the burden of recurrent and chronic digital ulcers in systemic sclerosis: long-term results from the DUO Registry. Ann Rheum Dis. 2016;75:1770-6.

[3] Allanore Y, Denton CP, Krieg T, etal.Clinical characteristics and predictosof gangrene in patients with systemic sclerosis and digital ulcers in Digital Ulcer Outcome Registry: a prospective, observational cohort. Ann Rheumatic Dis 2016;75:1736-1740.

Disclosure of Interest: None declared

DOI: 10.1136/annrheumdis-2017-eular.4828

\section{AB0663 THE RELATIONSHIP OF SERUM LEVEL OF ANTICCP ANTIBODY WITH SEVERITY OF SKIN INVOLVEMENT IN SYSTEMIC SCLEROSIS PATIENTS REFERRED TO TOHID HOSPITAL}

N. Moghimy ${ }^{1}$, A. Saeidi ${ }^{2} .{ }^{1}$ Liver and Digestive Research Center; ${ }^{2}$ Master of clinical Psychologist, Kordestan Medical Scienses, Sanandaj, Iran, Islamic Republic Of

Background: Systemic sclerosis is a chronic systemic disease of unknown etiology. There are a number of autoantibodies that correlated with disease severity and severity of skin involvement (1). Various studies have shown that AntiCCP Ab can also be seen in other autoimmune diseases such as systemic sclerosis (2-4). Results Polimen et al (4) and Morita et al (3) studies showed no relationship was found between AntiCCP Ab with skin Rodnon score.

Objectives: This study aimed to determine the relationship between serum levels of AntiCCP Ab with severity of skin involvement in systemic sclerosis patients referred to Tohid Hospital.

Methods: This study is a cross - sectional study, in the study population included all patients diagnosed with systemic sclerosis according to ACR 1980 criteria and subtypes according to Le Roy, that between 2013 and 2014 referred to Tohid hospital. We used from MRSS index to determine the extent of skin involvement. Sampling was available and we used to determine this association from chi-square statistics and to determine the amount of the connection from Phi statistics. Serum antibodies directed against CCP were assessed by ELISA

Results: The results showed that the patients who were evaluated at the end of study were 50 patients, of whom 48 were women (96\%) and (2\%) 2 were male. The mean age of subjects is $38 \pm 10$ years. The subtype of systemic sclerosis in 15 (\%30 percent) of the patients were diffuse systemic sclerosis and 35 patients (\%70 percent) have limited systemic sclerosis. Serum Anti CCP Abntibody was positive in $10 \%$ (5 patients). Also, there is no statistically significant relationship between the severity of skin involvement with Anticcp Ab $(p=0.164)$.Severity of skin involvement in both groups was more severe form, and there is no statistically significant relationship between the severity of skin involvement and subtypes of systemic sclerosis $(p=0.233)$. Also we didn't find no association between levels AntiCCP and joint symptoms and other symptoms.

Conclusions: In this study wasn't observed a significant correlation between serum levels of Anti CCP Antibody with severity of skin involvement in patients with systemic sclerosis $(p=0.164)$, also serum level of AntiCCP Antibody couldn't as a predict factor to determine the severity of the disease. It seems that the relationship between other symptoms of systemic sclerosis and serum level of AntiCCP Antibody may be result from overlapping with RA.

References:

[1] Shand L, Lunt M, Nihtianovas, Hoseini M, Silman A, Blake M "Relationship between Change in skine score and disease outcome in diffuse cutaneous scleroderma" arthritis rheum 2007 jul:56(7):2422-31.

[2] Marrone M, Chiala A, Tampoia M, lannone F et al, Prevalence of anti-CCP antibodies in systemic sclerosis, Reumatismo. 2007 Jan-Mar;59(1):20-4.

[3] Morita Y, Moro Y, Sugiura K, Tomita Y "Anti cyclic citrollinated Peptide in Systemic Sclerosis". 2008 jul:26(4):542-7.

[4] Polimeni M, Feniman D, Skare Ts, Nishihara Km "Anti cyclic citrollinated Peptide antibodies in scleroderma patints".clin rheumatal 2012 may;31(5):877-80.

Disclosure of Interest: None declared

DOI: 10.1136/annrheumdis-2017-eular.1159

\section{AB0664 LEVEL OF SATISFACTION IN PATIENTS WITH IDIOPATHIC INFLAMMATORY MYOPATHIES}

N. Loghin-Oprea ${ }^{1}$, S. Vetrila ${ }^{2}$, L. Mazur-Nicorici ${ }^{2}$, M. Cebanu ${ }^{3}$, V. Salaru ${ }^{4}$, M. Mazur ${ }^{1} .{ }^{1}$ Internal Medicine; ${ }^{2}$ Cardiology; ${ }^{3}$ Emergency Medicine; ${ }^{4}$ Family Medicine, PI SUMPh Nicolae Testemitanu, Chisinau, Moldova, Republic of

Background: Idiopathic Inflammatory Myopathies (IIM) are the group of rare diseases that carry a significant impact on patient's quality of life, influenced by the level of patient's satisfaction regarding medical services.

Objectives: To assess the patient's satisfaction and quality of life.

Methods: A cross-sectional study was performed from December 2015 to December 2016. There were included consecutive patients that fulfilled the Bohan and Peter ${ }^{1}$ criteria for IIM. The collected information was about demographic data, clinical and laboratory findings. The patient's satisfaction was assessed by self-administered Patient Satisfaction Questionnaire (PSQ III) ${ }^{2}$, which is a 50 -item tool, covering 7 domains: general satisfaction, technical quality, 
interpersonal aspects, communication, financial aspects, time spent with doctor, access/availability/convenience. To estimate the quality of life we applied Short Form-8 questionnaire with 8 items for 8 domains and two components: mental and physical.

Results: There were 32 patients enrolled in the study, including 23 females and 9 males, mean age $52.5 \pm 14.26$ (range $25-78$ ), the disease duration was $8.3 \pm 5.3$ (range $0.5-12$ ) years. The physical component was lower $37.49 \pm 8.49$, than the mental component $44.96 \pm 6.24$ points, we determined that the quality of patient's life was reduced. The PSQ III results were: general satisfaction $20 \pm 2.93$, technical quality $36.8 \pm 5.93$, interpersonal aspects $26.50 \pm 4.92$, communication $19.7 \pm 3.25$ and access/availability/convenience indicated $44.75 \pm 6.29$ score. The time spent with doctor was $7 \pm 1.15$, a border score. The average result in the financial aspects domain was $21.6 \pm 6.6$, lower than the midpoint scoring (24). We identified correlation between the quality of life and the access/availability/convenience PSQ III domain. For the mental compound the correlation was moderate $(r=0.5$ $p<0.005)$ and for physical domain it was weak $(r=0.27 p<0.005)$, these results can be explained by the fact that patients got accustomed with the disease, but they are more interested in access to care and require increasement of the duration of medical consultation.

Conclusions: Patients with idiopathic inflammatory myopathies have reduced quality of life; however they are satisfied of the medical attendance. The dissatisfaction was with the financial aspect, due to the long-term disease course and high indirect costs.

\section{References:}

[1] Bohan A, Peter JB. N Engl J Med 1975 Feb 13;292(7):344-7.

[2] Ware J.Jr. et al. Defining and measuring patient satisfaction with medical care. Eval Program Plann. 1983; 6(3-4): 247-63.

Disclosure of Interest: None declared

DOI: 10.1136/annrheumdis-2017-eular.4844

\section{AB0665 ASSOCIATION BETWEEN MICROVACULATURE CHANGES AND PULMONARY INVOLVEMENT IN SYSTEMIC SCLEROSIS: A FOLLOW-UP STUDY}

R. Bečvár ${ }^{1}, M$. Tomčík ${ }^{2}$, J. Štork ${ }^{3}, A$. Slováková ${ }^{4} .{ }^{1}$ Department of Rheumatology; ${ }^{2}$ Institute of Rheumatology; ${ }^{3}$ Department of Dermatovenerology; ${ }^{4}$ Department of Pneumology, General Teaching Hospital, Praha 2, Czech Republic

Background: Lung involvement is the major cause of mortality in patients with systemic sclerosis (SSc). Gas transfer (DLCO) and FVC levels have traditionally been used as measures of disease severity and reductions of both parameters have been associated with increased mortality. In SSc repeated attacks of Raynaud's phenomenon lead to the reduced capillary density leads with reduced blood flow and tissue ischemia. Raynaud's phenomenon can occur also in the lungs. Tissue hypoxia usually initiates the formation of new blood vessels from the pre-existing microvasculature. Nailfold capillaroscopy is a safe, noninvasive routine way for the microvascular investigation.

Objectives: The aim of this study was to assess the correlation between capillaroscopic abnormalities and parameters of interstitial lung involvement at baseline and after one year follow-up in patients with SSc.

Methods: All patients underwent routine clinical examination (dyspnea, cough, crepitus), pulmonary function tests, DLCO (alveolitis grade and fibrosis), blood gases and HRCT scan of chest (1). Microvasculature changes were assessed using nailfold videocapillaroscopy (NVC) which was performed by two independent examiners. The obtained images were analysed anonymously by two investigators blinded for the clinical and serum status of SSc patients and classified as early, active and late pattern, non specific or normal picture (2). For statistical evaluation Poisson's correlation coefficient and T-test were used. All examinations were performed at baseline and after 12 months.

Results: Total 42 patients ( 38 females) were investigated: 30 individuals with limited form, 7 with diffuse form, 3 patients with scleroderma sine scleroderma, 1 with overlap syndrome and 1 with undifferentiated connective tissue disease. The mean age \pm standard deviation (SD) of the whole cohort was $51 \pm 22$ years and the mean disease duration \pm SD was $10 \pm 7$ years. 3 patients $(7.5 \%)$ had early NVC pattern, 12 patients $(30 \%)$ had active, $10(58 \%)$ late pattern, and 17 $(37.5 \%)$ had nonspecific changes or normal picture. The patients with late NVC pattern exhibited more often mild to moderate alveolitis, decreased FEV1 and FVC and DLCO. When correlating NVC patterns with clinical findings, pulmonary function test and HRCT scans we found only an association of low significance with dyspnea and alveolitis grade (both $p<0.1$ ). After one year follow-up similar results were obtained $(p<0,001$ and $p<0,01$, respectively).

Conclusions: In our study NVC patterns did not seem to correlate with severity of interstitial lung disease in SSc patients even after one year follow-up. References:

[1] Corrado A et al. Joint Bone Spine. 2010.

[2] Cutolo M et al. J Rheumatol. 2000.

Acknowledgements: This study was performed with support of $\mathrm{CMH}$ Research

Projects No 00000023728 .

Disclosure of Interest: None declared

DOI: 10.1136/annrheumdis-2017-eular.2421

\section{AB0666 MYOPATHIES ASSOCIATED WITH SYSTEMIC SCLEROSIS: CLINICAL AND IMMUNOLOGICAL FEATURES FROM A TERTIARY CENTRE COHORT}

R. Nichitean, A. Mociran, I. Szabo, L. Damian, I. Felea, S. Siao-Pin, S. Rednic. Rheumatology, Scju Cluj Napoca, Cluj Napoca, Romania

Background: Systemic sclerosis (SSc) is a connective tissue disease characterized by skin and internal organ fibrosis due to immune activation, vascular damage and excessive synthesis of collagen [1]. Muscle involvement is still a variable feature, with prevalence from $14 \%$ to $79 \%$ due to the lack of diagnostic consensus criteria [2].

Objectives: The aim of this study was to characterize the clinical, biochemical and immunological features of SSc patients with muscle involvement and to find any correlations amongst them.

Methods: We retrospectively analysed data for 18 SSc patients with myopathy out of 254 SSc patients followed between 2000 and 2016 in a Romanian academic hospital centre. All patients fulfilled the ACR/EULAR criteria for the diagnosis of SSc and had a history of myopathy. Myopathy was defined as the presence of muscle weakness, myalgia, with creatine kinase (CK) and/or lactate dehydrogenase (LDH) level $>2 \mathrm{~N}$ (two times the upper normal range). Clinical, biochemical and immunological parameters were assessed by descriptive statistics, logistic regression and Chi-square test.

Results: The prevalence of myopathy was $7.0 \%$. General features in myopathySSc patients are detailed in Table 1. Male sex represents a risk factor for muscle involvement $(\mathrm{OR}=38.2, p$-value $<0.001)$. The ratio of diffuse subtype to the limited subtype was 0.8 . The major organ involvement associated with myopathy was lung fibrosis (55.5\%). Most prevalent autoantibody was anti-Scl70 $(44.4 \%)$. The onset of muscle symptoms occurred within a median of 1.6 years (range 0-12 years) after SSc diagnosis. Among the studied patients, 12 (66.6\%) had muscle weakness, and $13(72.2 \%)$ had myalgia. CK and LDH levels were elevated in $16(88.88 \%)$ and $18(100 \%)$ cases, respectively. AST level values were found to be slightly higher than ALT ones but not statistically significant ( $p$-value $=0.15$ ). A positive correlation was found between levels of LDH and AST (Pearson coefficient $=0.72$ ) and between $\mathrm{CK}$ and LDH (Pearson coefficient $=0.66$ ). No association was identified with inflammatory parameters.

Table 1.

\begin{tabular}{|c|c|c|}
\hline \multicolumn{2}{|c|}{ SSc general data and associated features } & Number out of 18 (total) and \% \\
\hline \multicolumn{2}{|l|}{ Female sex } & $12(66.6 \%)$ \\
\hline \multicolumn{2}{|l|}{ Diffuse SSc form } & $8(44.4 \%)$ \\
\hline \multicolumn{2}{|c|}{ Age at SSc diagnosis } & 43 ye ars \\
\hline \multicolumn{2}{|c|}{ Digital ulcers } & $3(16.6 \%)$ \\
\hline \multicolumn{2}{|l|}{ Calcinosis } & $6(33.3 \%)$ \\
\hline \multirow[t]{5}{*}{ Autoantibodies } & Anti-nuclear & $16(88.8 \%)$ \\
\hline & Anti-Scl70 & $8(44.4 \%)$ \\
\hline & Anti-centromere & 0 \\
\hline & Anti-RNP & $2(11.1 \%)$ \\
\hline & Anti-PM/Scl & $1(5.5 \%)$ \\
\hline \multicolumn{2}{|c|}{ Heart involvement } & $2(11.1 \%)$ \\
\hline \multicolumn{2}{|l|}{ Lung fibrosis } & $10(55.5 \%)$ \\
\hline \multicolumn{2}{|c|}{ Scleroderma renal crisis } & $2(11.1 \%)$ \\
\hline \multicolumn{2}{|l|}{ Arthritis } & $4(22.2 \%)$ \\
\hline \multicolumn{2}{|c|}{ Oesophagus involvement } & $6(33.3 \%)$ \\
\hline \multicolumn{2}{|l|}{ Motor weakness } & $12(66.6 \%)$ \\
\hline \multicolumn{2}{|l|}{ Myalgia } & $13(72.2 \%)$ \\
\hline
\end{tabular}

Conclusions: This study emphasizes the fact that male SSc patients are at increased risk of developing myopathy. Although the prevalence of muscle involvement was quite low than reported in other cohort series, the results are similar to the more recent publications [3]. We showed that myopathy occurred more often in anti-Scl70 positive patients and that anti-centromere wasn't present at all. All patients should be carefully assessed for muscle involvement in the first years of SSc diagnosis and screened for lung fibrosis, regardless of cutaneous subtype of the disease.

\section{References:}

[1] Systemic sclerosis: current pathogenetic concepts and future prospects for targeted therapy. Lancet 1996; 347:1453.

[2] Paik, J., Mammen, A., Wigley, F. and Gelber A. Myopathy in scleroderma, its identification, prevalence, and treatment: lessons learned from cohort studies. Curr Opin Rheumatol 26: 124-130.

[3] RaB Ranque, A Bérezné, V Le-Guern, C Pagnoux et al. Myopathies related to systemic sclerosis: a case-control study of associated clinical and immunological features. Scand J Rheumatol 2010;39:498-505.

Disclosure of Interest: None declared

DOI: 10.1136/annrheumdis-2017-eular.1579 